\title{
Application of mild pulsed electric fields on starter culture accelerates yogurt fermentation
}

\author{
Panagiotis Chanos $^{1}$ (1) $\cdot$ Malou C. Warncke ${ }^{2} \cdot$ Matthias A. Ehrmann $^{2} \cdot$ Christian Hertel $^{1}$
}

Received: 14 October 2019 / Accepted: 31 December 2019 / Published online: 20 January 2020

(c) The Author(s) 2020

\begin{abstract}
The goal of this work was to investigate the influence of pulsed electric fields (PEF) operational parameters on a mixture of Streptococcus thermophilus DIL 5218 and Lactobacillus delbrueckii subsp. bulgaricus DSMZ $20081^{\mathrm{T}}$ with regards to the culture's acidification capability in reconstituted skim milk medium. We investigated the effects of field strength, pulse frequency and total number of pulses by use of design of experiments and a two-level full factorial design. The responses were the cell counts of the two microorganisms after PEF application, the $\mathrm{pH}$ lag phase $\lambda_{\mathrm{pH}}$, the maximum $\mathrm{pH}$ change rate $\mu_{\max }$, the maximum $\mathrm{pH}$ change and the oxidation/reduction potential (ORP). The application of PEF on the mixed culture accelerated the acidification of milk by an average of $12 \mathrm{~min}$ in an approx. $160 \mathrm{~min}$ lasting control $\lambda_{\mathrm{pH}}$. In contrast the maximum $\mathrm{pH}$ change rate $\mu_{\max }$ and the maximum $\mathrm{pH}$ change decreased slightly in fermentations with PEF-treated cultures. Furthermore, a significantly faster decrease of the oxidation/reduction potential (ORP) already within the first $30 \mathrm{~min}$ and a lower final ORP was observed in milk fermented with PEF-treated culture. The total number of pulses applied was the most influencing factor in most of the responses measured. We hypothesized that the reason for the enhanced performance of the PEF-treated culture was a combination of an oxidative stress response of S. thermophilus DIL 5218 and an enhanced proteolytic phenotype in L. delbrueckii subsp. bulgaricus DSMZ $20081^{\mathrm{T}}$.
\end{abstract}

Keywords Pulsed electric fields $\cdot$ Starter culture $\cdot$ Yogurt $\cdot$ Acidification acceleration $\cdot$ Stress response

\section{Introduction}

The commercial production of set yogurt is aided with the use of special starter cultures of S. thermophilus and L. delbrueckii subsp. bulgaricus. The associative growth of the two-termed proto-cooperation-has been shown to lead to higher acidification rates and lower end-fermentation $\mathrm{pH}$ than in single strain fermentations [1,2]. Furthermore higher S. thermophilus cell density [3] and higher production of aroma compounds and exopolysaccharides $[4,5]$ have been observed. The rapid acidification in the fermentation of milk serves mainly two purposes: the safety of the final product by inhibition of pathogens through the rapid production of

Panagiotis Chanos

p.chanos@dil-ev.de

1 Department of Biotechnology, German Institute of Food Technologies (DIL), Professor-von-Klitzing-Str. 7, 49610 Quakenbrueck, Germany

2 Technical Microbiology, Technical University of Munich, Gregor-Mendel Str., 85354 Freising, Germany lactic acid and the development of the gel network through the aggregation of casein as the $\mathrm{pH}$ drops to the caseins' isoelectric point $[6,7]$. In the industrial production of yogurt, upon addition of the starter culture to milk a $\mathrm{pH}$ lag phase [8] is seen where cultures adjust to the new milk environment and where no $\mathrm{pH}$ change is observed. A short $\mathrm{pH}$ lag phase is of high interest to the industry, to shorten the fermentation time under the condition that no adverse effects on the sensory characteristics of the yogurt occur.

Pulsed electric fields (PEF) are a novel technology, among others, for the pasteurization of liquid foods. In comparison to pasteurization, PEF have the ability to decontaminate foods without a considerable increase in their temperature, thus, preserving their sensory characteristics. The technology is based on the delivery of a pulsing power between the electrodes of a treatment chamber containing the liquid food [9]. The electrical pulses are conducted through the food to the membrane of the microbial cell causing electroporation and under certain intensities irreversible cell membrane damages, leakage of intracellular material and cell death. The effectiveness of microbial inactivation depends on the 
microbial characteristics, the treatment medium characteristics as well as several PEF operational factors. These are the electric field strength, the treatment time, the frequency of the pulses the specific energy applied as well as the geometry and the polarity of the pulses [10]. The application of PEF for the pasteurization of liquid food has been successful in various products depending on the amount of total energy applied [11]. However, when the total energy applied by PEF is reduced to sublethal levels it can lead to a growthstimulating effect [12] or to reversible electroporation of the membrane. Technologically significant effects of sublethal PEF on food-grade microorganisms included the increase in viability and fermentation activity of Saccharomyces cerevisiae [13-15] and the induction of increased growth rate, acid tolerance, proteolytic activity and bacteriocin formation in Lactobacillus spp. [16-18].

The aim of this study was to evaluate whether the application of PEF on yogurt starter culture influences its acidification capability and whether this effect could be influenced by the levels of the individual PEF-related parameters like field strength, pulse frequency and number of pulses applied.

\section{Materials and methods}

\section{Cell suspension preparation}

S. thermophilus DIL 5218 and L. delbrueckii subsp. bulgaricus DSMZ $20081^{\mathrm{T}}$ were used as yogurt starter culture and routinely cultured in M17 medium (Oxoid) and MRS medium (Carl Roth) at $37{ }^{\circ} \mathrm{C}$ and $42{ }^{\circ} \mathrm{C}$, respectively. Cells counts were determined using M17 agar (Oxoid) and MRS agar (Carl Roth) and incubating at $37^{\circ} \mathrm{C}$ and $42{ }^{\circ} \mathrm{C}$, respectively, for $24 \mathrm{~h}$. For each experiment the strains were revived from frozen glycerol stocks on agar plates. Overnight liquid cultures were prepared by inoculating a colony in the corresponding liquid medium and incubating for $20 \mathrm{~h}$. Working cultures were prepared by inoculation of the liquid medium with $10 \%$ overnight liquid culture and incubation at the relevant temperature for $4 \mathrm{~h}$ until reaching the middle of the exponential phase. Cell suspensions were prepared by centrifugation of a working culture at $10,000 \times g$ and washing once in $0.1 \%$ peptone water (Carl Roth) before re-suspending in the same medium. The desired cell concentration of $7.5 \times 10^{7} \mathrm{CFU} / \mathrm{mL}$ was achieved by adding sterile $0.1 \%$ peptone water.

\section{PEF treatment of cell suspensions}

The treatment of the cell suspensions of $S$. thermophilus DIL 5218 and L. bulgaricus DSMZ $20081^{\mathrm{T}}$ was done in an
ELCRACK HPV Batch PEF equipment (Elea Vertriebs- und Vermarktungsgesellschaft mbH, Quakenbrück, Germany) with a capacitor of $0.5 \mu \mathrm{F}$. The treatment chamber had a volume of $125 \mathrm{~mL}$ and was composed of two stainless steel parallel electrodes separated from each other with white Teflon insulation of $3 \mathrm{~cm}$ length. The energy was delivered to the samples in the form of exponential decay pulses. The effects of three factors, namely, $X 1$ : the field strength, $X 2$ : the pulse frequency and $X 3$ : the total number of pulses on the acidification capacity of the cultures were evaluated by the use of a $2^{3}$ full factorial arrangement resulting in 8 runs per experiment (Table 1). The specific energy applied in each run was calculated using Eq. 1.

$E=0.5 \cdot C \cdot U^{2} \cdot n$

where $C$ is the capacitance, $U$ is the voltage used and $n$ is the number of pulses. The corresponding values of each factor for both levels are shown in Table 2. PEF treatments of the mixed culture were done in biological quadruplicates and each replicate represented a completely randomized block.

\section{Fermentations}

Duplicate fermentation bottles were inoculated with cell suspension of each run resulting in a total of 16 fermentation bottles per experiment. All fermentations were done in $250 \mathrm{~mL}$ Schott bottles with round openings on the lids to allow exactly the insertion of the $\mathrm{pH}$ electrode. The lids were fitted with rubber gaskets to prevent the introduction of condensed water in the flask. In each flask, $245 \mathrm{~mL}$ of $9 \%(w / v)$ sterilized $\left(121^{\circ} \mathrm{C}, 15 \mathrm{~min}\right.$ ) skim milk powder (DMK GmbH, D-Zeven) was used as fermentation medium. The medium was tempered to $42{ }^{\circ} \mathrm{C}$ before being inoculated with $5 \mathrm{~mL}$ of either PEF-treated or not treated cell-suspension and mixed well by repeated aspirating and dispensing using a serological pipette. The final theoretical concentration of untreated cells in the milk was $1.5 \times 10^{5} \mathrm{CFU} / \mathrm{mL}$. The incubation of the inoculated skim milk bottles was done in a water bath (LAUDA Eco RE 2025) at $42{ }^{\circ} \mathrm{C}$ and the $\mathrm{pH}$ and the oxidation reduction potential (ORP) development was followed by an iCinac analyzer system (AMS Alliance, F-Frépillon) for $480 \mathrm{~min}$. The positions of the bottles in the water bath were fully randomized to avoid possible deviations introduced from the proximity of the bottles to the heating element of the water bath. Two flasks with milk medium were inoculated with PEF-treated $\mathrm{pH}$ cell suspension from each PEF run. Each fermentation was repeated 4 times (equal to the number of PEF-treated biological replicates). Control fermentations with not PEF-treated cell-suspension mix were 




Table 2 Levels of independent factors used

\begin{tabular}{lll}
\hline Levels & -1 & 1 \\
\hline Field strength $\left(\mathrm{kV} \mathrm{cm}^{-1}\right)$ & 1 & 3.67 \\
Frequency $(\mathrm{Hz})$ & 0.5 & 4 \\
Number of pulses & 5 & 50 \\
\hline
\end{tabular}

repeated twice and each repeat consisted of 6 fermentation bottles randomly positioned in the water bath. As $t_{0}$ was considered the timepoint $5 \mathrm{~min}$ after the inoculation of the milk medium with cell suspension.

For each run the relative $\mathrm{pH}$-change of each fermentation flask to $t_{0}$ was plotted against time. The XLfit ${ }^{\circledR}$ (IDBS) MSExcel add-in was used to fit the Gompertz model (Eq. 2) to the absolute $\mathrm{pH}$ change values between 0.2 and 1.2 against time and to calculate parameters $a, b$ and $c$. The $\mathrm{pH}$ lag phase $(\lambda)$ and maximum $\mathrm{pH}$ change rate $\left(\mu_{\max }\right)$ were calculated by Eqs. 3 and 4, respectively, as derived from the modified Gompertz equation (Eq. 4) [8].

$y=a \cdot \exp [-\exp (b-c x)]$

$\lambda=(b-1) / c$

$\mu_{\mathrm{m}}=(c \cdot a) / e$

$y=a \cdot \exp -\exp \left[\left(\mu_{\mathrm{m}} \cdot e\right) / a(\lambda-t)+1\right]$

where $\alpha$ is the value for the asymptote $(y=\alpha)$ and $b$ and $c$ set the displacement of the graph on the $\mathrm{x}$ axis and the growth rate, respectively. For each run the $\lambda_{\mathrm{pH}}$ was calculated as the mean of the $\lambda_{\mathrm{pH}}$ values of each fermentation flask $(n=2)$.

The counts of the surviving cells (SC) were evaluated by agar plating after each PEF treatment in six replicates. Earlier experiments had shown that differential enumeration of the $L$. bulgaricus DSMZ $20081^{\mathrm{T}}$ and $S$. thermophilus DIL 5218 cell counts in a mixed culture was possible by plating on MRS agar (Oxoid) at $42{ }^{\circ} \mathrm{C}$ anaerobically and on M17 agar (Oxoid) aerobically at $37{ }^{\circ} \mathrm{C}$, respectively. For enumeration of not-injured cells (NI) $\mathrm{NaCl}$ was used as stressor at different concentrations in the respective agars for each strain. The enumeration of the NI cell counts was also done in six replicates. The threshold concentration of $\mathrm{NaCl}$ in the media used for the detection of NI for L. bulgaricus DSMZ $20081^{\mathrm{T}}$ and for S. thermophilus DIL 5218 cells was $1.9 \%(w / v)$ and $1.1 \%(w / v)$, respectively, as shown in earlier experiments (ANOVA, Tukey's post-hoc test, $p<0.05$, results not shown). The statistical analysis was done with MINITAB $18^{\circledR}$ statistical software, version 18.1. 


\section{Results}

\section{Counts of surviving and not-injured cells}

Based on the cell counts determined after PEF treatment of the starter culture and depending on the different PEF conditions applied, an average of $15.4 \%$ of the calculated initial inoculum $\left(7.5 \times 10^{7} \mathrm{CFU} / \mathrm{mL}\right)$ of $S$. thermophilus DIL 5218 and $24.3 \%$ of that of L. bulgaricus DSMZ $20081^{\mathrm{T}}$ survived the treatment (Table 1). Furthermore, $86.2 \%$ of the surviving S. themophilus cells were not injured as they managed to grow on the stress medium containing $\mathrm{NaCl}$. In contrast, an average of only $64.8 \%$ of the surviving $L$. bulgaricus cells could be characterized as not injured. From the factorial regression analysis, it was concluded that only the parameter field strength exhibited a significant effect $(p=0.014$, $\alpha=0.05$ ) on the counts of SC of L. bulgaricus. Increase in the field strength led to lower SC counts.

\section{Effect of PEF parameters on $\lambda$ and $\mu_{\max }$ of the starter culture}

The coefficient of determination $\left(R^{2}\right)$ of the models fitted to the data was $>0.998$ for all fermentations. Application of PEF on the starter culture increased its acidification capability compared to a fermentation with not PEF-treated culture. Depending on the PEF conditions, a decrease of the $\mathrm{pH}$ lag phase $\lambda_{\mathrm{pH}}$ of up to 12 min was observed (Fig. 1).
The calculated means of $\lambda_{\mathrm{pH}}(n=4)$ in relation to different PEF conditions and compared to the $\lambda_{\mathrm{pH}}$ of fermentations done with not PEF-treated starter cultures are listed in Table 1. Analysis of variance of the $\lambda_{\mathrm{pH}}$ of the different treatment groups and the control did not reveal statistically significant differences $(p>0.05)$. As depicted in the multivari chart in Fig. 2 the combination of high number of pulses (right panel), high frequency (right part of the right panel) and low field strength (triangle pointing down) tended to cause the lowest $\lambda_{\mathrm{pH}}$, as also shown in Table 1 .

The factorial regression analysis of the response $\lambda_{\mathrm{pH}}$ for the mixed yogurt culture indicated that the number of pulses and the interaction of number of pulses and frequency had a significant effect on the $\mathrm{pH}$ lag phase $(p=0.04$ and $p=0.000$, respectively). High number of pulses, low field strength as well as the combination of high frequency and high number of pulses tended to minimize $\lambda_{\mathrm{pH}}$. The lineal regression model fitted to the response $\lambda_{\mathrm{pH}}$ had a coefficient of determination $\left(R^{2}\right)$ of 0.911 and the adjusted $R^{2}$ for the number of predictors (factors) was 0.8728 , indicating that the model could explain $87.28 \%$ of the variability of the response. No apparent violation of the assumptions for ANOVA was observed.

Interestingly, the mean $\mu_{\max }$ in the fermentations where PEF-treated cultures were used tended to be equal or lower than that of the fermentations done with not-PEF-treated cultures (Table 1). Analysis of variance of the $\mu_{\max }$ of the different treatment groups and the control, however, did not exhibit statistically significant differences $(p>0.05)$.
Fig. 1 Absolute $\mathrm{pH}$ change of milk medium inoculated with not PEF-treated starter culture (open circles) and with PEFtreated culture (run 3 ) with 50 pulses at $4 \mathrm{~Hz}$ and $1 \mathrm{kV} / \mathrm{cm}$ field strength (closed circles) during an incubation at $42{ }^{\circ} \mathrm{C}$ for $480 \mathrm{~min}$




Fig. 2 Muti-vari chart for $\lambda_{\mathrm{pH}}$, showing the gradients across all fermentations tested. Panel variable: number of pulses; the left and right panels represent low and high number of pulses, respectively. Rhombuses detail the mean $\lambda_{\mathrm{pH}}$ calculated at each pulse number level tested. Squares detail the mean $\lambda_{\mathrm{pH}}$ at each pulse number-frequency combination tested. Pyramids and inverted pyramids detail field strength, respectively, at each pulse number-frequency combination tested the mean $\lambda_{\mathrm{pH}}$ at high and low

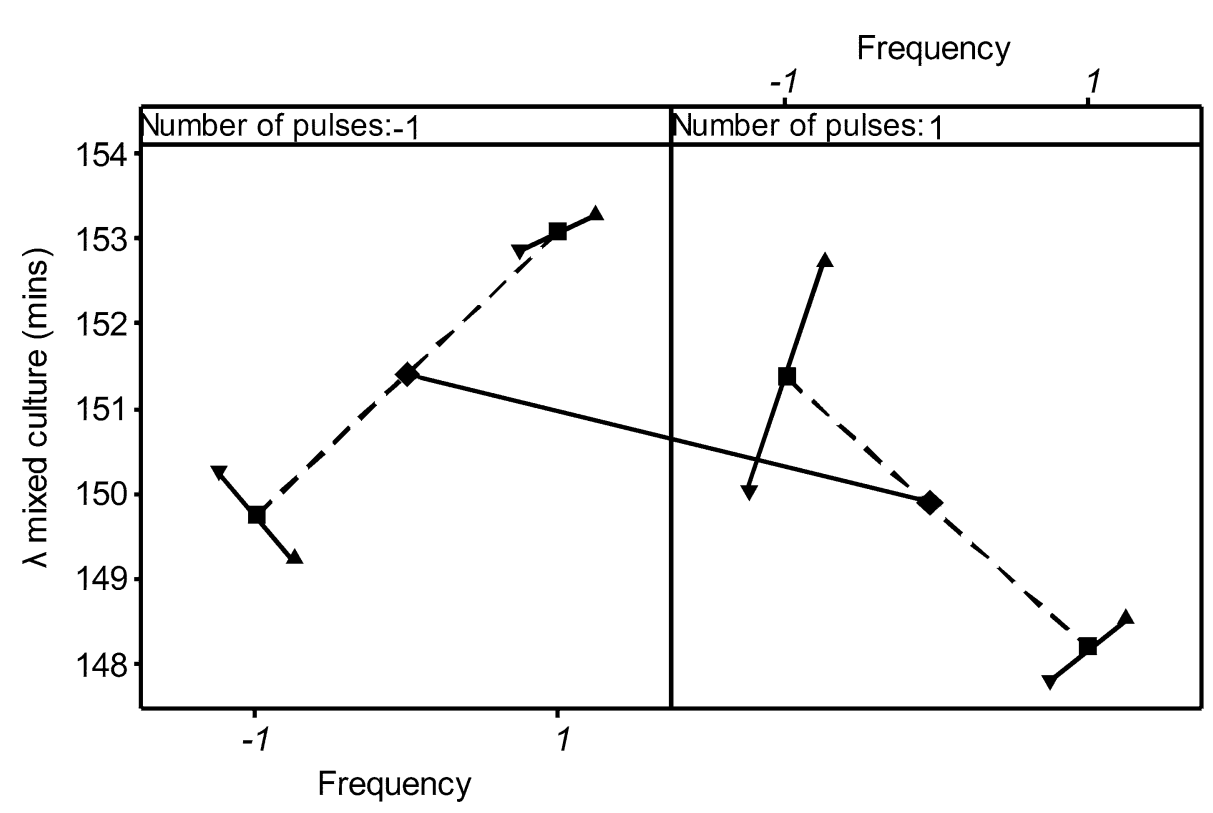

Fig. 3 Muti-vari chart for $\mu_{\max }$, showing the gradients across all fermentations tested. Panel variable: number of pulses; the left and right panels represent low and high number of pulses, respectively. Rhombuses detail the mean $\mu_{\max }$ calculated at each pulse number level tested. Squares detail the mean $\mu_{\max }$ at each pulse number-frequency combination tested. Pyramids and inverted pyramids detail the mean $\mu_{\max }$ at high and low field strength, respectively, at each pulse number-frequency combination tested

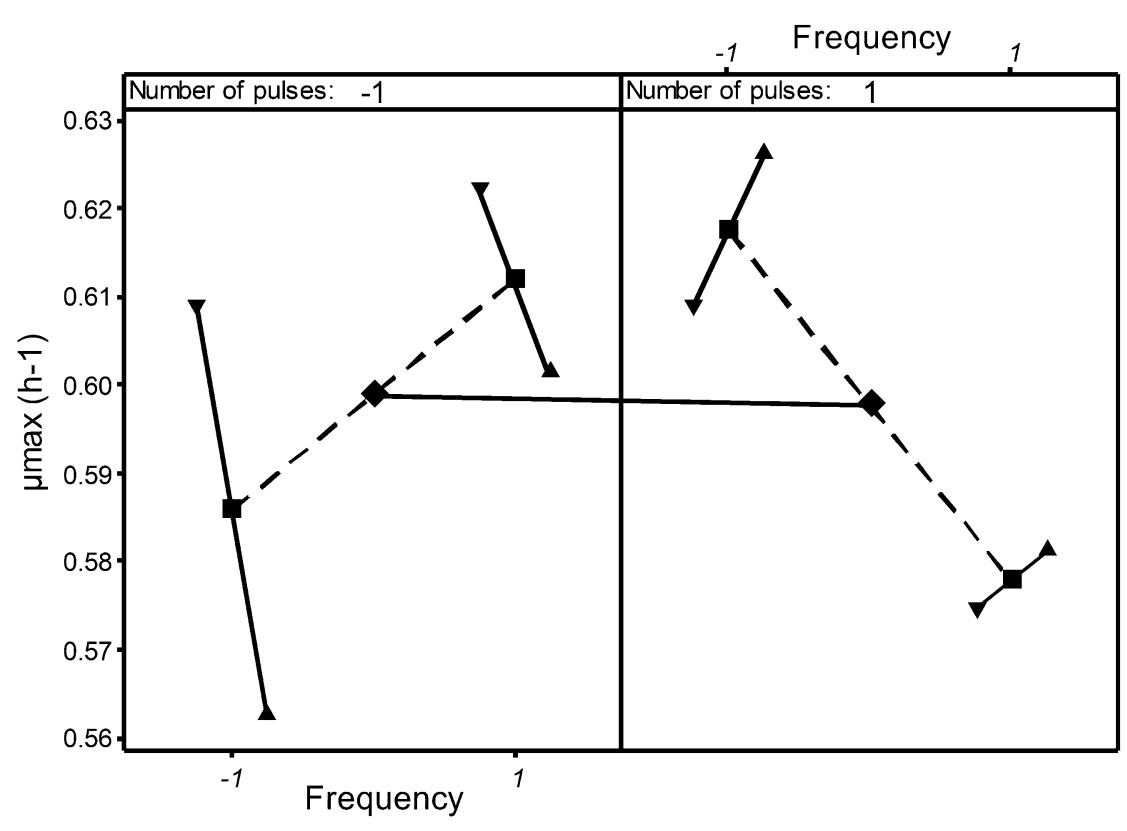

Factorial regression analysis of the $\mu_{\max }$ in the fermentations with PEF-treated mixed culture revealed that the interaction of number of pulses and the frequency of the pulses had a significant effect $(p=0.02)$ on $\mu_{\max }$. When a low number of pulses was applied ( 5 pulses), increase of the frequency from 0.5 to $4 \mathrm{~Hz}$ tended to increase the mean $\mu_{\text {max }}$. On the contrary, at high number of pulses (50 pulses) an increase in the frequency from $(0.5-4 \mathrm{~Hz})$ tended to decrease the mean $\mu_{\text {max }}$ (Fig. 3).

The $R^{2}$ for the linear regression model fitted to the response $\mu_{\max }$ was 0.509 , the adjusted $R^{2}$ for the number of predictors (factors) was 0.3085 . Analysis of the standardized residuals indicated that they were normally distributed, had a constant variance and were independent from each other. One observation with a high standardized residual was found. The deviation of this point from the model could not be explained and therefore further analysis was done to evaluate its influence on the model. Exclusion of this observation from the analysis or transformation of the data and new regression analysis were attempted. No change in the result was observed regardless of the methodology followed (data not shown). 


\section{Effect of PEF parameters on oxidation reduction potential}

Treatment of the mixed culture with PEF resulted in a faster reduction of the oxidation reduction potential (ORP) of the milk in comparison to a milk fermentation with non-PEFtreated cultures. The accelerated reduction of the ORP was evident already in the first $5 \mathrm{~min}$ of the fermentation, as it can be seen in Fig. 4. Application of PEF at high field strength $(3.67 \mathrm{kV} / \mathrm{cm})$, low frequency $(0.5 \mathrm{~Hz})$ and high pulse number (50 pulses) resulted on average in the fastest decrease of ORP (run 7) while treating the culture with the same field strength and frequency but with low number of pulses ( 5 pulses) resulted in the slowest ORP decrease (run 1). Comparison of the absolute decrease of ORP at 2, 10 and $30 \mathrm{~min}$ of fermentation showed that there was a statistically significant difference between the mean absolute ORP decrease of run 7 and that of the fermentations done with not PEF-treated mixed culture (Student's test, $p<0.05$ ). The difference indicated that the number of pulses was playing an important role in the enhancement of the culture's ability to decrease the redox potential.

Factorial regression analysis of the mean absolute ORP decrease in the first $30 \mathrm{~min}$ of incubation revealed that frequency $(p=0.021)$ and the interaction of frequency and number of pulses $(p=0.005)$ had a statistically significant impact on the redox potential decrease $\left(R^{2}=0.637\right.$, $\left.R_{\text {adj. }}^{2}=0.465\right)$. No apparent violation of the assumptions for ANOVA was observed. Analysis of variance of the different treatment groups did not reveal statistically significant differences $(p>0.05)$. When applying low number of pulses, increases in frequency from 0.5 to $4 \mathrm{~Hz}$ tended to cause a faster decrease of the ORP regardless of the field strength applied. When applying high number of pulses, however, the ORP decrease depended on the combination of field strength and frequency (Fig. 5). At high field strength $(3.7 \mathrm{kV} / \mathrm{cm})$ an increase in the frequency led to smaller ORP decreases in the fermented milk while at low field strength an increase in the frequency led to higher ORP decreases.

\section{Effect of the PEF parameters in the maximum $\mathrm{pH}$ change}

Application of PEF on the culture had a negative effect on the maximum $\mathrm{pH}$ change within the $480 \mathrm{~min}$ fermentation time as compared to the maximum $\mathrm{pH}$ change achieved with a not PEF-treated mixed culture in the same time. Factorial regression analysis showed that the number of pulses $(p=0.006)$ and the field strength $(p=0.034)$ had a significant effect on the maximum $\mathrm{pH}$ decrease $\left(R^{2}=0.841\right.$, $\left.R_{\text {adj. }}^{2}=0.765\right)$. No apparent violation of the assumptions for ANOVA was observed. Analysis of variance of the maximum $\mathrm{pH}$ change in the different treatment groups did not reveal statistically significant differences $(p>0.05)$. Higher number of pulses and low field strength tended to cause greater decrease of $\mathrm{pH}$ as seen in Fig. 6.
Fig. 4 Development of the mean absolute ORP during the fermentation of milk medium at $42{ }^{\circ} \mathrm{C}$ for $480 \mathrm{~min}$. Runs $1-8$ correspond to fermentations done with the mixed starter culture treated with different PEF conditions

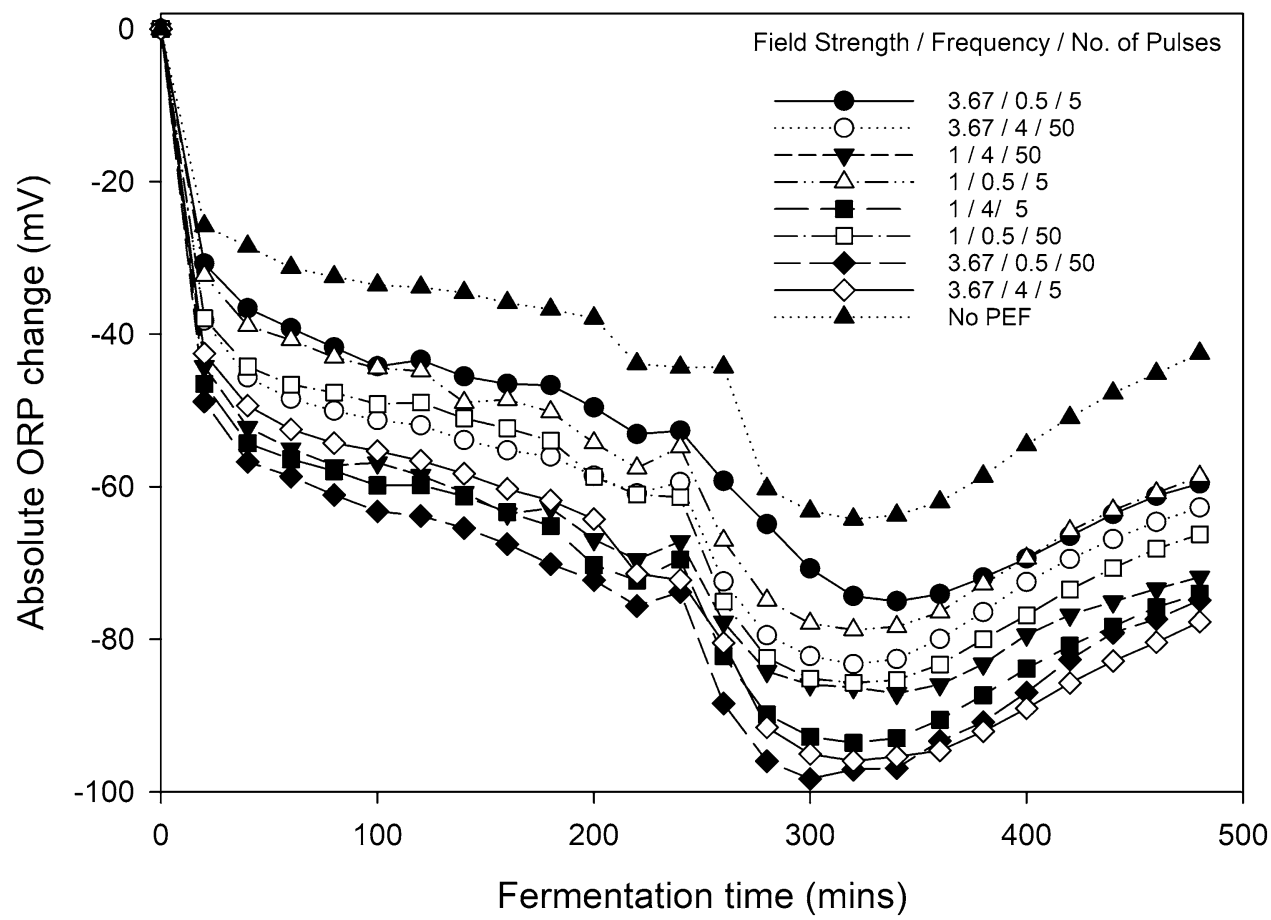


Fig. 5 Muti-vari chart for absolute ORP change, showing the gradients across all fermentations tested. Panel variable: number of pulses; the left and right panels represent low and high number of pulses, respectively. Rhombuses detail the mean absolute ORP change calculated at each pulse number level tested. Squares detail the mean absolute ORP change at each pulse number-frequency combination tested. Pyramids and inverted pyramids detail the mean absolute ORP change at high and low field strength, respectively, at each pulse number-frequency combination tested
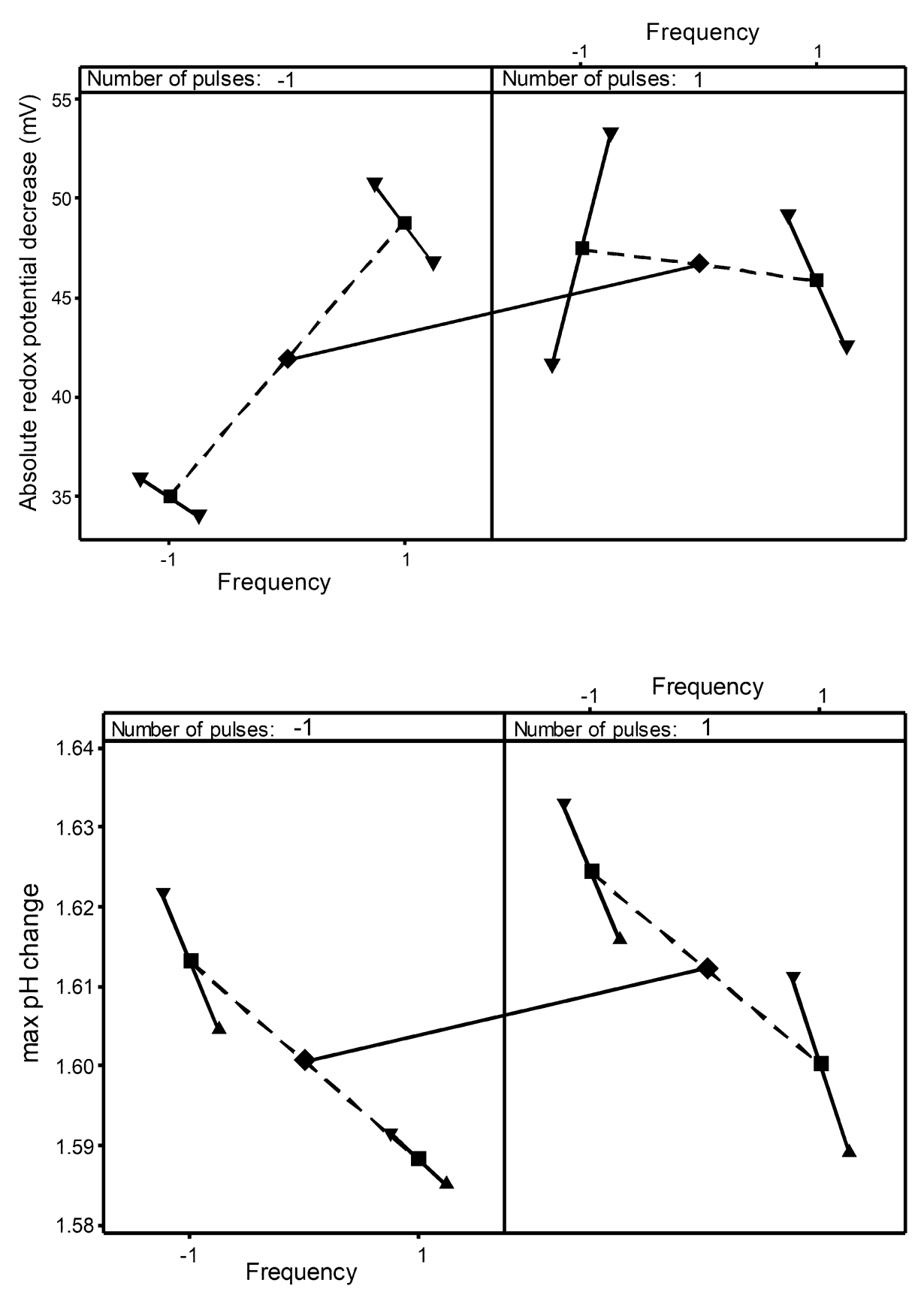

Fig. 6 Muti-vari chart for max. $\mathrm{pH}$ change, showing the gradients across all fermentations tested. Panel variable: number of pulses; the left and right panels represent low and high number of pulses, respectively. Rhombuses detail the mean max. $\mathrm{pH}$ change calculated at each pulse number level tested. Squares detail the mean max. $\mathrm{pH}$ change at each pulse number-frequency combination tested. Pyramids and inverted pyramids detail the mean max. $\mathrm{pH}$ change at high and low field strength, respectively, at each pulse number-frequency combination tested

\section{Discussion}

In this work we influenced the development of $\mathrm{pH}$ and ORP in the fermentation of milk medium by applying, in advance, pulsed electric fields on yogurt starter cultures. Interestingly an acceleration of the acidification and a drastic reduction of the ORP were achieved with lower initial inocula compared to the control fermentation, as application of PEF on the starter culture led to a more than $50 \%$ reduction of the initial inoculum size and the production of sub-lethally injured cells in the populations of both starter strains.
The stimulating effect of the application of electrical current (direct current, alternating current or short pulses) on the metabolism of microorganisms has been documented for the growth and metabolic processes of different microorganisms, as recently reviewed by Mota et al. [19]. Although the mechanism by which this effect can be explained is not yet fully elucidated, two possible mechanisms have been suggested:

a. The formation of pores by electroporation and increased diffusion of ions and molecules which is augmenting nutrient uptake leading to increased performance [20]. 
b. Stimulation of metabolic cascades and control of metabolic pathways because of the activity of electric fields [21].

Electropermeabilization of cells with PEF has been shown to cause an osmotic imbalance because of the loss of $\mathrm{Na}+, \mathrm{K}+$, or other cytoplasmic substances and swelling of the cells due to increased intake of solution from the cell's environment [22]. Enhanced nutrient uptake due to this inwards positive flux has been considered as the possible cause of increased performance in cells treated with mild electric fields [23]. For example, decrease in the growth lag phase in fermentations with L. acidophilus due to the application of mild electric fields $(1 \mathrm{~V} / \mathrm{cm})$ has been shown earlier [18]. The lag phase duration was positively correlated with the frequency of the current used, i.e., lower frequencies (45 Hz and $60 \mathrm{~Hz}$ ) caused shorter lag phase durations. The authors associated this effect with increased mass transfer due to the formation of electropores, as already shown for eukaryotic cells [24], although increase in mass transfer was found to be associated with increasing voltages [18]. In our experiments, the peptone water solution used for application of PEF on the culture cells, contained a pancreatic digest of casein which is rich in amino acids and peptides essential for growth. Massive transfer of essential nutrients inside the cells of the culture may have induced a rapid kick-off of metabolic processes giving a head start to the PEF-treated culture compared to the untreated one. However, in contrast to the findings of Loghavi et al. [18], in our experiments shorter lag phases tended to correlate with lower field strength (lower voltage), indicating that nutrient transfer by electroporation is less likely to be the underlying cause for this performance increase observed.

Alternatively, since transfer of solutes caused by electroporation of a membrane is bidirectional, as shown previously by the presence of nucleic acids in the environment of electroporated cells [22], a stress response due to pore formation resulting in leakage of intracellular material might also cause increase in the metabolism. Leakage of intracellular material including small molecules, like ATP and cations because of pore formation, is a usual result of the action of antimicrobial peptides like bacteriocins. In this aspect, application of stress on Listeria monocytogenes $08-5923$ by exposure to sublethal doses of carnocyclin A, a bacteriocin from Carnobacterium maltaromaticum has been shown to induce the upregulation of genes encoding metabolismrelated proteins like the pyruvate kinase PykA [25].

The application of pulsed electric fields at sublethal levels for the cells has been known to induce oxidative stress responses [26]. Indeed, high voltage discharge in water as well as cells in aqueous suspensions causes the formation of reactive oxygen species (ROS) like $\mathrm{H}_{2} \mathrm{O}_{2}$ not only in the medium but also inside the cells [27, 28]. Most of lactic acid bacteria have been shown to possess a multitude of stress-induced response mechanisms involving the activation of several metabolic processes to protect themselves from heat, cold, oxidative and other shocks [29]. However, L. delbrueckii subsp. bulgaricus lacks the genetic determinants to synthesize detoxification enzymes like catalases, peroxidases or superoxide dismutases [30]. Therefore, the presence of $\mathrm{H}_{2} \mathrm{O}_{2}$ in the medium or intracellularly would cause a retardation and not an acceleration of its metabolism [31]. S. thermophilus on the contrary has multiple response mechanisms to detoxify from ROS including $\mathrm{H}_{2} \mathrm{O}$-forming NADH oxidases, NADH peroxidases, superoxide dismutases as well as a generalized antioxidant potential through metal ion chelating [32]. Furthermore random insertional mutagenesis studies in $S$. thermophilus CNRZ368 revealed that expression of the same seven genes was involved in the stress response of the microorganism to different oxidative stress inducers including oxygen (as dissolved oxygen in liquid medium), hydrogen peroxide and methyl viologen [33]. In set yogurt production, the dissolved oxygen concentration is likely to be high in the beginning of the fermentation due to the incorporation of oxygen through the mixing employed to distribute the starter culture in the milk mass.

Moreover, it has been shown that $S$. thermophilus accelerates L-lactate and formate accumulation in fermentation when the milk is deoxygenated [34]. It is therefore conceivable that in our work the application of sublethal levels of PEF on S. thermophilus DIL 5218 elicited an oxidative stress response in the cells and thus, allowed them to quickly adapt to the oxidative environment of the oxygenated milk medium. Indeed, our experimental results showed that ORP of fermentations with PEF-treated starter culture exhibited a rapid decrease compared to the control fermentation and remained significantly lower throughout the fermentation. The frequency of pulses applied to the culture seemed to play a significant role in the development of the ORP. Especially when a total of 50 pulses were applied on the culture at $3.7 \mathrm{kV} / \mathrm{cm}$ field strength, an increase in the frequency from 0.5 to $4 \mathrm{~Hz}$ caused a rise in the ORP values (lower oxidative effect). Pakhomova et al. [28] showed a similar effect of pulse frequency and field strength in the creation of ROS in a medium. Interestingly, when 50 pulses of nano-second PEF were applied in cell-free medium at $9.8 \mathrm{kV} / \mathrm{cm}$ field strength, an increase of frequency from 0.3 to $1 \mathrm{~Hz}$ caused a reduction of the oxidative effect. Assuming that, within reasonable limits, higher concentration of ROS induces a greater stress response and in turn a steeper decrease in the ORP in milk medium, these results confirm our findings.

The response to the oxidative stress and the strong reduction of ORP though cannot fully explain the acceleration of the fermentation observed with some PEF treatments. Looking closer to the runs with the strongest ORP reduction, it can be seen that they are also among the ones with 
the slowest fermentation in terms of $\lambda_{\mathrm{pH}}$. In contrast, run 3 exhibited the shortest mean $\lambda_{\mathrm{pH}}$ and also one of the steepest ORP reduction among the fermentations done with PEFtreated cultures. In our experiments the counts of L. bulgaricus DSMZ $20081^{\mathrm{T}}$ were significantly affected $(p<0.05)$ only by the field strength applied, in the sense that higher field strength resulted in lower NI cell counts. In run 3 the counts of NI cells are among the highest in the PEF-treated samples. A possible explanation to that could be that the presence of L. bulgaricus DSMZ $20081^{\mathrm{T}}$ acted as an amplifier of the acidification effect, since it is known that formate produced by S. thermophilus induces the growth of L. delbrueckii subsp. bulgaricus [35]. This in return may have stimulated the growth of $S$. thermophilus by production of small peptides using its strong proteolytic machinery [36, 37]. Indeed, application of mild PEF treatment $(1 \mathrm{kV} / \mathrm{cm})$ on L. delbrueckii subsp. bulgaricus has been shown to significantly increase its proteolytic activity [17] which further supports this argument.

To our knowledge, this is the first study to show the successful use of PEF in improving the performance of lactic acid bacteria in food fermentation. We showed that application of mild PEF treatment on dairy starter cultures, under specific conditions, can cause an earlier onset of $\mathrm{pH}$ decrease in the fermentation which may be a desirable characteristic for the industry. Based on this information, further research is needed to characterise the effect of the PEF parameters within a broader range of values on the metabolism of the starter organisms. The application of PEF on starter cultures for yogurt manufacture is bound to elicit responses at the transcriptome level, possibly manifested in the phenotype of the microorganisms. The study of the possible relations between the different PEF parameters, the microorganisms' genomes and transcriptomes and their phenotypical responses as well as their connection to the development of the sensory characteristics of yogurt, could open a new field of applied research.

Acknowledgements Open Access funding provided by Projekt DEAL.

\section{Compliance with ethical standards}

Conflict of interest The authors declare no conflict of interest.

Compliance with Ethics requirements Neither human nor animal rights were violated in this work.

Open Access This article is licensed under a Creative Commons Attribution 4.0 International License, which permits use, sharing, adaptation, distribution and reproduction in any medium or format, as long as you give appropriate credit to the original author(s) and the source, provide a link to the Creative Commons licence, and indicate if changes were made. The images or other third party material in this article are included in the article's Creative Commons licence, unless indicated otherwise in a credit line to the material. If material is not included in the article's Creative Commons licence and your intended use is not permitted by statutory regulation or exceeds the permitted use, you will need to obtain permission directly from the copyright holder. To view a copy of this licence, visit http://creativecommons.org/licenses/by/4.0/.

\section{References}

1. Amoroso MJ, Manca de Nadra MC, Oliver G (1989) The growth and sugar utilization by Lactobacillus delbrueckii ssp. bulgaricus and Streptococcus salivarius ssp. thermophilus isolated from market yogurt. Lait 69:519-528. https://doi.org/10.1051/lait:1989635

2. Beal C, Corrieu G (1991) Influence of pH, temperature, and inoculum composition on mixed cultures of Streptococcus thermophilus 404 and Lactobacillus bulgaricus 398. Biotechnol Bioeng 38:9098. https://doi.org/10.1002/bit.260380112

3. Rajagopal SN, Sandine WE (1990) Associative growth and proteolysis of Streptococcus thermophilus and Lactobacillus bulgaricus in skim milk. J Dairy Sci 73:894-899. https://doi.org/10.3168/ jds.S0022-0302(90)78745-0

4. Courtin P, Rul F (2004) Interactions between microorganisms in a simple ecosystem: yogurt bacteria as a study model. Lait 84:125-134. https://doi.org/10.1051/lait:2003031

5. Bouzar F, Cerning J, Desmazeaud M (1997) Exopolysaccharide production and texture-promoting abilities of mixed-strain starter cultures in yogurt production. J Dairy Sci 80:2310-2317. https:// doi.org/10.3168/jds.S0022-0302(97)76181-2

6. Damin MR, Alcântara MR, Nunes AP, Oliveira MN (2009) Effects of milk supplementation with skim milk powder, whey protein concentrate and sodium caseinate on acidification kinetics, rheological properties and structure of nonfat stirred yogurt. LWT Food Sci Technol 42:1744-1750. https://doi.org/10.1016/j. lwt.2009.03.019

7. Rubin HE, Vaughan F (1979) Elucidation of the inhibitory factors of yogurt against Salmonella typhimurium. J Dairy Sci 62:18731879. https://doi.org/10.3168/JDS.S0022-0302(79)83517-1

8. Zwietering MH, Jongenburger I, Rompouts FM, Van't Riet K (1990) Modeling of the bacterial growth curve. Appl Environ Microbiol 56:1875-1881

9. Barbosa-Cánovas GV, Altunakar B (2006) Pulsed electric fields processing of foods: an overview. In: Raso J, Heinz V (eds) Pulsed electric fields technology for the food industry: fundamentals and applications. Springer, Boston, pp 3-26

10. Álvarez I, Condón S, Raso J (2006) Microbial inactivation by pulsed electric fields. In: Raso J, Heinz V (eds) Pulsed electric fields technology for the food industry: fundamentals and applications. Springer, Boston, pp 97-129

11. Toepfl S, Heinz V, Knorr D (2006) Applications of pulsed electric fields technology for the food industry. In: Raso J, Heinz V (eds) Pulsed electric fields technology for the food industry, 1st edn. Springer, New York, pp 197-221

12. Frey W, Flickinger B, Berghoefer T, et al (2011) Electropermeabilization versus nsPEF-stimulation-pulsed electric fields can stimulate the growth of plants and fungi. In: Proceedings of the 10th international conference of the european bioelectromagnetic association, Roma, pp 21-24

13. Mattar JR, Turk MF, Nonus M et al (2014) Stimulation of Saccharomyces cerevisiae cultures by pulsed electric fields. Food Bioprocess Technol 7:3328-3335. https://doi.org/10.1007/s1194 7-014-1336-4

14. Mattar JR, Turk MF, Nonus M et al (2015) S. cerevisiae fermentation activity after moderate pulsed electric field pre-treatments. Bioelectrochemistry 103:92-97. https://doi.org/10.1016/j.bioel echem.2014.08.016 
15. Castro I, Oliveira C, Domingues L et al (2012) The effect of the electric field on lag phase, $\beta$-galactosidase production and plasmid stability of a recombinant Saccharomyces cerevisiae strain growing on lactose. Food Bioprocess Technol 5:3014-3020. https:// doi.org/10.1007/s11947-011-0609-4

16. Seratlić S, Bugarski B, Nedović V et al (2013) Behavior of the surviving population of Lactobacillus plantarum 564 upon the application of pulsed electric fields. Innov Food Sci Emerg Technol 17:93-98. https://doi.org/10.1016/j.ifset.2012.11.011

17. Najim N, Aryana KJ (2013) A mild pulsed electric field condition that improves acid tolerance, growth, and protease activity of Lactobacillus acidophilus LA-K and Lactobacillus delbrueckii subspecies bulgaricus LB-12. J Dairy Sci 96:3424-3434. https:// doi.org/10.3168/jds.2012-5842

18. Loghavi L, Sastry SK, Yousef AE (2008) Effect of moderate electric field frequency on growth kinetics and metabolic activity of Lactobacillus acidophilus. Biotechnol Prog 24:148-153. https:// doi.org/10.1021/bp070268v

19. Mota MJ, Lopes RP, Koubaa M et al (2017) Fermentation at nonconventional conditions in food- and bio-sciences by the application of advanced processing technologies. Crit Rev Biotechnol. https://doi.org/10.1080/07388551.2017.1312272

20. Shil P, Bidaye S, Vidyasagar PB (2008) Analysing the effects of surface distribution of pores in cell electroporation for a cell membrane containing cholesterol. J Phys D Appl Phys 41:055502. https://doi.org/10.1088/0022-3727/41/5/055502

21. Hunt R, Zavalin A, Bhatnagar A et al (2009) Electromagnetic biostimulation of living cultures for biotechnology, biofuel and bioenergy applications. Int J Mol Sci 10:4515-4558. https://doi. org/10.3390/ijms10104515

22. Pillet F, Formosa-Dague C, Baaziz H et al (2016) Cell wall as a target for bacteria inactivation by pulsed electric fields. Sci Rep 6:1-8. https://doi.org/10.1038/srep19778

23. Tryfona T, Bustard MT (2008) Impact of pulsed electric fields on Corynebacterium glutamicum cell membrane permeabilization. $\mathrm{J}$ Biosci Bioeng 105:375-382. https://doi.org/10.1263/jbb.105.375

24. Kulshrestha S, Sastry S (2003) Frequency and voltage effects on enhanced diffusion during moderate electric field (MEF) treatment. Innov Food Sci Emerg Technol 4:189-194. https://doi. org/10.1016/S1466-8564(03)00003-1

25. Liu X, Basu U, Miller P, McMullen LM (2014) Stress response and adaptation of Listeria monocytogenes 08-5923 exposed to a sublethal dose of Carnocyclin A. Appl Environ Microbiol 80:3835-3841. https://doi.org/10.1128/AEM.00350-14

26. Wang M-S, Wang L-H, Bekhit AE-DA et al (2018) A review of sublethal effects of pulsed electric field on cells in food processing. J Food Eng 223:32-41. https://doi.org/10.1016/j.jfood eng.2017.11.035
27. Sato M, Ohgiyama T, Clements JS (1996) Formation of chemical species and their effects on microorganisms using a pulsed highvoltage discharge in water. IEEE Trans Ind Appl 32:106-112. https://doi.org/10.1109/28.485820

28. Pakhomova ON, Khorokhorina VA, Bowman AM et al (2012) Oxidative effects of nanosecond pulsed electric field exposure in cells and cell-free media. Arch Biochem Biophys 527:55-64. https ://doi.org/10.1016/j.abb.2012.08.004

29. Papadimitriou K, Alegría Á, Bron PA et al (2016) Stress physiology of lactic acid bacteria. Microbiol Mol Biol Rev 80:837-890. https://doi.org/10.1128/MMBR.00076-15

30. van de Guchte M, Penaud S, Grimaldi C et al (2006) The complete genome sequence of Lactobacillus bulgaricus reveals extensive and ongoing reductive evolution. Proc Natl Acad Sci 103:92749279. https://doi.org/10.1073/pnas.0603024103

31. Marty-Teysset C, de la Torre F, Garel J-R (2000) Increased production of hydrogen peroxide by Lactobacillus delbrueckii subsp. bulgaricus upon aeration: involvement of an NADH oxidase in oxidative stress. Appl Environ Microbiol 66:262-267. https://doi. org/10.1128/AEM.66.1.262-267.2000

32. Lemos JA, Tsakalidou E, Papadimitriou K (2011) Stress responses of Streptococci. Stress responses of lactic acid bacteria. Springer, Boston, pp 251-303

33. Fernandez A, Thibessard A, Borges F et al (2004) Characterisation of oxidative stress-resistant mutants of Streptococcus themophillus CNRZ368. Arch Microbiol 182:364-372. https://doi. org/10.1007/s00203-004-0712-2

34. Sasaki Y, Horiuchi H, Kawashima H et al (2014) NADH oxidase of Streptococcusthemophillus 1131 is required for the effective yogurt fermentation with Lactobacillus delbrueckii subsp. bulgaricus 2038. Biosci Microbiota Food Health 33:31-40. https://doi. org/10.12938/bmfh.33.31

35. Galesloot TE, Hassing F, Veringa HA (1968) Symbiosis in yoghurt. I. Stimulation of Lactobacillus bulgaricus by a factor produced by Streptococcus themophillus. Neth Milk Dairy J 22:50 (Nederlands melk en zuivelltijdschrift)

36. Moon NJ, Reinbold GW (1976) Commensalism and competition in mixed cultures of Lactobacillus bulgaricus and Streptococcus themophillus. J Milk Food Technol 39:337-341. https://doi. org/10.4315/0022-2747-39.5.337

37. Mihail A, Kostov G, Angelov M et al (2009) Proto-cooperation factors in yogurt starter cultures. Rev Génie Ind 3:4-12

Publisher's Note Springer Nature remains neutral with regard to jurisdictional claims in published maps and institutional affiliations. 